\title{
Traumatic Posterior Sternoclavicular Joint Dislocation in a 20 Year Old Male: A Case Report
}

\author{
Leanne McKeown PA-C ${ }^{1}$ and Mitesh K Patel MD ${ }^{2 *}$ \\ ${ }^{1}$ Rothman Orthopaedic Institute, USA \\ ${ }^{2}$ Department of Orthopaedics, Rothman Orthopaedic Institute, Sports Medicine, \\ Thomas Jefferson University, USA \\ *Corresponding Author: Mitesh K Patel, Department of Orthopaedics, Rothman \\ Orthopaedic Institute, Sports Medicine, Thomas Jefferson University, USA.
}

Received: January 27, 2021

Published: February 16, 2021

(C) All rights are reserved by Leanne

McKeown and Mitesh K Patel.

\begin{abstract}
Sternoclavicular posterior joint dislocations are rare and typically occur from a high-energy injury. The author reports the case of a 20-year-old male who sustained a left sternoclavicular posterior dislocation after wrestling with his brother. He presented with persistent left shoulder pain and swelling at an Orthopaedic Specialty Urgent care after discharge from an emergency room with a sling and soft collar. Standard chest radiographs were normal. Chest CT scan demonstrated posterior dislocation of the occipital head with sharp posterior edge impinging on the proximal aortic arch's anterior wall. The patient underwent a sternoclavicular joint ORIF with suture anchor repair. After four months, the patient reports a resolution of pain and mobility. The patient's injury was initially undiagnosed, emphasizing the importance of recognizing this rare event and obtaining a CT scan for early diagnosis.
\end{abstract}

Keywords: Sternoclavicular Joint Dislocation; CT Scan; Posterior Dislocation

\section{Background}

SCJ dislocations comprise $1 \%$ of all dislocations and $3 \%$ of upper limb dislocations. [1] Direct force on the medial clavicle can cause a type 3 rupture of the SCJ and costoclavicular ligament that forces the clavicle to be pushed posteriorly into the mediastinum [1]. In patients between the ages of 23 and 35, a fracture of the medial physis should be considered as this area is last to ossify [2]. Posterior dislocation and fracture are associated with mediastinal injuries, which may be life-threatening [2]. The objective is to discuss the mechanism and diagnosis in an often-misdiagnosed injury.

\section{Case Presentation}

A 20-year-old auto mechanic was wrestling with his brother, who wrapped his arms around him from behind, and they both fell to the floor onto his left shoulder. He felt immediate pain in his left shoulder with a pop in his left sternoclavicular area. He had increasing shoulder pain and difficulty raising his arm. He presented to a hospital emergency room for evaluation. The pain was $8 / 10$ on a visual analog scale. Vitals were BP 132/86, HR 73, Resp 20, Sat $96<$ temp 98.2. The patient denied weakness or numbness. Radiographs were read as normal, and the patient was discharged with a shoulder sling and soft collar.

He presented the next day to an Orthopaedic Urgent care for evaluation due to persistent pain. He complained of an increase in shoulder and new onset of anterior chest pain, and pain with deep breath and cough. He denied dysphagia, odynophagia, dyspnea, hoarseness and choking.

On exam, the patient was 6' tall and weighed $165 \mathrm{lbs}$ with a BMI of 22.38. Inspection demonstrated swelling over the left sterno- 
clavicular joint without ecchymosis. Sensation was intact to light touch. The distal radial pulse was intact and equal. There was a palpable defect on the left clavicle near the sternal end and focal tenderness at the SC joint. Left shoulder range of motion was decreased due to pain across his left clavicle and chest.

Two view X-rays of the bilateral clavicle and three-view left shoulder X-ray demonstrated no fracture, dislocation, or radiopaque foreign body. The underlying ribs intact are intact (Figure $1)$.

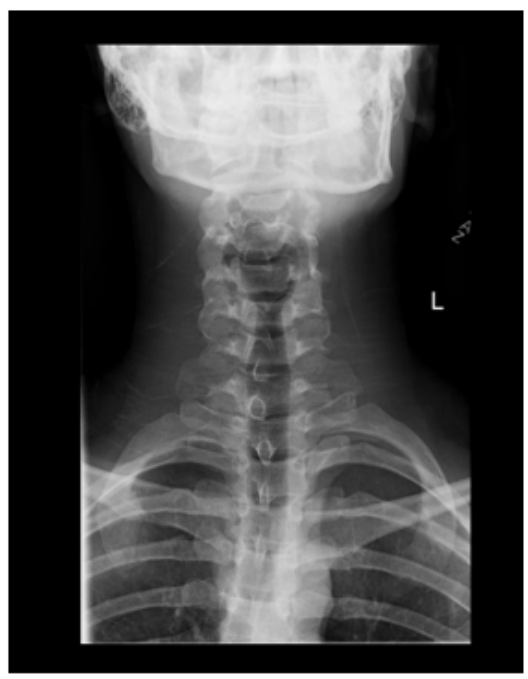

Figure 1

The diagnosis for posterior SCJ dislocation was suspected. The patient was sent for a Computed Tomography to better visualize any bony or soft tissue injury and tissues within the mediastinum. The CT scan confirmed complete posterior dislocation of the left clavicle's proximal head, noting a relatively sharp edge of the proximal metaphysis of the clavicular head abutting the anterior wall of the proximal aortic arch (Figure 2-6).

The patient was transported immediately from the radiology suite to the emergency room, where Orthopaedics and Thoracic surgery were consulted. Vitals remained stable at BP 108/80, HR 55, Resp 10, Sat 99, Temp 98.2. CTA chest w/wo contrast was performed, which showed a displaced fragment that indents the left
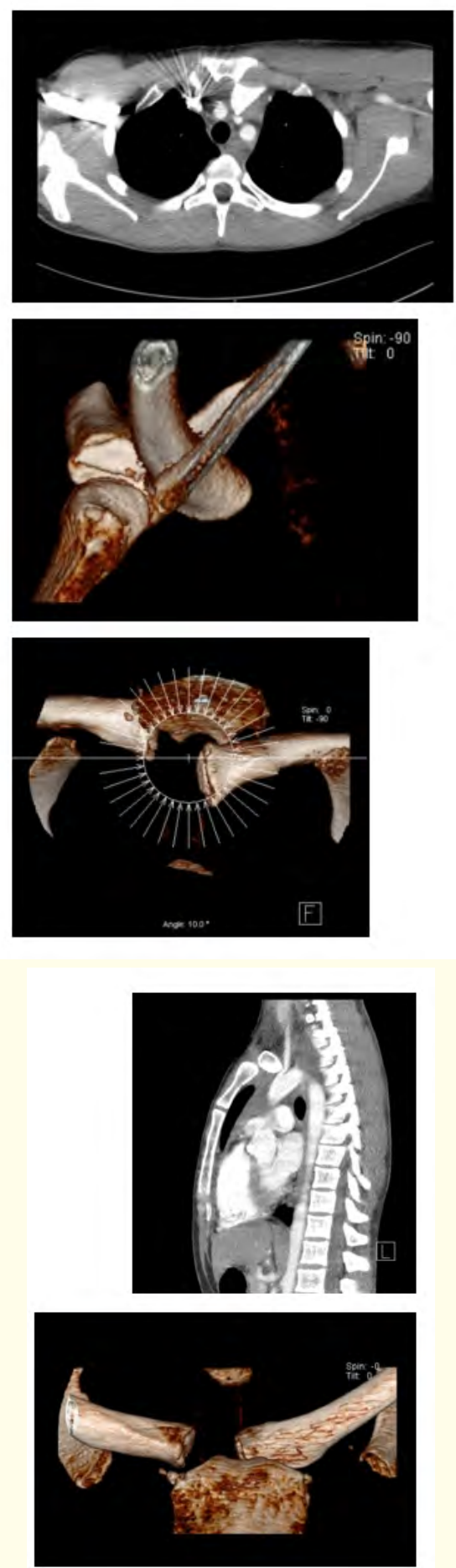

Figure 2-6 
common carotid artery and brachiocephalic vein with mild/moderate narrowing with no arterial contrast extravasation. Small pericardial effusion was noted (Figure 7 and 8).
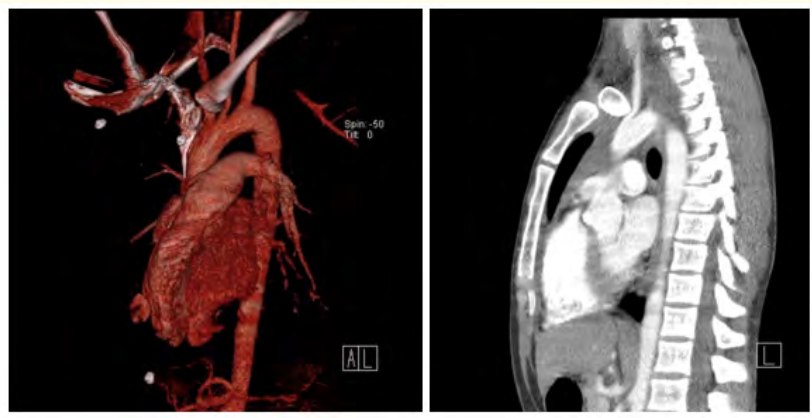

Figure 7 and 8

Upper extremity venous Doppler Ultrasound was performed, which demonstrated compressible internal jugular and subclavian vein with spontaneous and pulsatile flow without thrombosis. The patient was transferred to a tertiary Level 1 trauma hospital for surgical intervention. On arrival, vital remained stable with pain at $7 / 10$. The patient underwent left sternoclavicular joint ORIF using suture anchors without complications. Thoracic surgery was available during the procedure but ultimately was not needed. The patient was discharged the following day with weight-bearing precautions and limited UE ROM (Figure 9).

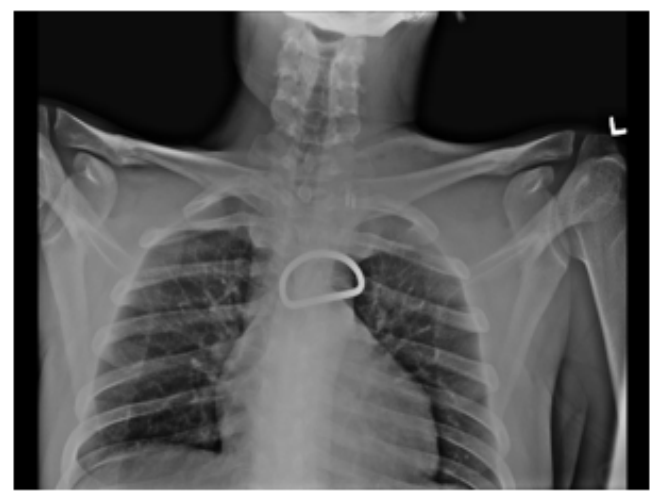

Figure 9

\section{Discussion and Conclusion}

SCJ injuries may be overlooked in a traumatic high-energy setting in which other injuries may take precedence [3]. However, posterior SCJ dislocation and fractures pose a significant risk and potentially life-threatening injuries [3]. The medial clavicle can be pushed posteriorly into the mediastinum with enough force [1]. Thirty percent of patients who sustain a posterior SCJ dislocation sustain a mediastinal injury, which can block venous flow from clavicle impingement, most commonly the brachiocephalic vein [3]. Impingement can present as dysphagia, cough, hoarseness, and choking sensation [3]. Patients can also develop upper extremity swelling, decreased range of motion, and new-onset paresthesia [2]. A weakened pulse is a sign of venous congestion from clavicle compression. Patients often present with the other arm supporting the injured arm and the patient's head tilting toward the injured shoulder [1]. Adolescent patients often have a medial physeal fracture, as the medial epiphysis does not fuse until between ages 23 - 25 [1]. The medial epiphysis is a weak point at this age during trauma, which can fracture and increase the risk of vascular laceration in addition to compression [1]. This injury must be recognized promptly as it can lead to high mortality and complications [2]. Radiographs are poor at diagnosing an SCJ injury and are often missed due to overlapping bones in this region [4]. Often, more severe injuries from the trauma are treated before recognition of an SCJ. A CT imaging study has become the test of choice if there is a high clinical suspicion [2]. CT scans can better evaluate the bony anatomy as well as identify potential mediastinal injuries [3]. Before surgical intervention, a CT angiograph was often ordered to access vascular injury and surgical planning [3]. Management of SCJ posterior dislocations may be treated by closed reduction or open reduction. In an acute setting, closed reduction may be achievable while under sedation or general anesthesia while under sedation or general anesthesia [5]. The injured shoulder should be positioned near the edge of the table while a 3- to 4 - inch think bolster is placed between the scapulae to extend the shoulders. Traction techniques are applied to the abducted arm while bringing it into extension. A sterile towel clip may be necessary to grasp the clavicle percutaneously to assist in reduction. An audible snap can be noted if the reduction is successful. Intraoperative radiographs should be performed to confirm an appropriate reduction [5]. When numerous potential complications make closed reduction too dangerous, then open surgical fixation 
is indicated. A thoracic surgeon should assist or be made immediately available during open reduction cases. In this case study, the thoracic surgeon was available but ultimately not needed to assist the Orthopaedic surgeon. The patient was placed in a supine position on the operative table, and the anesthesia team administered sedation. An inverted curvilinear line was made from the center of the manubrium to the medial clavicle $7 \mathrm{~cm}$ in length. Dissection was performed to the level of the clavipectoral fascia, where a rent was noted. The injury was noted to be through the physis medially. A clamp was placed on the clavicle, pulled anteriorly, causing reduction. Hemostasis was maintained during the procedure, and no vascular injury was observed. After reduction, two Mitek suture anchors were placed into the medial clavicle off the articular margin. The anterior sternoclavicular ligaments were repaired with 0 FiberWire suture and tied to prevent posterior displacement of the clavicle. Post-operative dressings were applied, and a sling was placed. The patient awoke from anesthesia without complication [op note]. Sternoclavicular injuries are rare but are associated with serious and life-threatening complications due to the close proximity to the hilar anatomy [5]. Careful physical examination to recognize a sternoclavicular posterior injury is imperative when evaluating a patient with a shoulder injury. Observation of a patient's body language and a higher level of pain may help differentiate a posterior SC injury from an anterior injury. The affected shoulder may appear shorter and subcutaneous swelling will be at the SC joint. Dyspnea, stridor, dysphagia, or paresthesia would suggest significant mediastinal injuries requiring emergent evaluation. A high suspicion of a posterior sternoclavicular dislocation warrants CT and examination by an Orthopaedic and Thoracic specialist [6]. Differential diagnosis should include sternum or rib fracture, infection (septic arthritis or osteomyelitis), Friedrich's disease, SAPHO (synovitis, acne, pustulosis, hyperostosis, and osteitis) syndrome, and multidirectional instability [6].

\section{Bibliography}

1. Dhawan R., et al. "Sternoclavicular joint". Shoulder Elbow 10.4 (2018): 296-305.

2. Morell DJ and Thyagarajan DS. "Sternoclavicular joint dislocation and its management: A review of the literature". World Journal of Orthopedics 7.4 (2016): 244-250.
3. Garcia JA., et al. "Sternoclavicular Joint Instability: Symptoms, Diagnosis And Management". Orthopedic Research and Reviews 12 (2020): 75-87.

4. Pinto A., et al. "Traumatic fractures in adults: missed diagnosis on plain radiographs in the Emergency Department". Acta Biomed 89.1 (2018): 111-123.

5. Groh Gordon I MD and Wirth Michael A MD "Management of Traumatic Sternoclavicular Joint Injuries". American Academy of Orthopaedic Surgeon 19.1 (2011): 1-7.

6. Kiel J., et al. "Sternoclavicular Joint Injury". In: StatPearls [Internet]. Treasure Island (FL): StatPearls Publishing (2020).

\section{Assets from publication with us}

- Prompt Acknowledgement after receiving the article

- Thorough Double blinded peer review

- Rapid Publication

- Issue of Publication Certificate

- High visibility of your Published work

Website: www.actascientific.com/

Submit Article: www.actascientific.com/submission.php

Email us: editor@actascientific.com

Contact us: +919182824667 